\title{
Efeitos de diferentes tipos de poda nos teores de nutrientes em flores e frutos de goiabeira
}

\author{
Differences among nutrients in flowers and fruits of guava trees \\ under different types of pruning \\ Henrique A. de Souza ${ }^{1 *}$, Danilo Eduardo Rozane ${ }^{2}$ Liliane Maria Romualdo $^{3}$, \\ William Natale 4
}

\section{RESUMO}

Um melhor conhecimento das diferenças entre os teores de nutrientes, em função do tipo de poda empregada na cultura da goiaba, pode permitir compreensão mais adequada dos processos fisiológicos nessa frutífera. A análise de flores é uma ferramenta que pode ser utilizada para auxiliar na avaliação do estado nutricional das culturas, especialmente as perenes. Assim, avaliaram-se os efeitos de diferentes tipos de poda nos teores de nutrientes em flores e frutos (em diferentes estádios de desenvolvimento e, em diferentes partes das goiabas). O estudo foi realizado em Vista Alegre do Alto, em pomares de goiabeiras 'Paluma'. Foram coletados flores e frutos em pomares distintos, sendo um deles conduzido sob poda drástica e outro sob poda contínua. Os frutos foram coletados em dois estádios (chumbinho e maduro) e divididos em parte proximal e distal, sendo a parte proximal voltada para o pedúnculo. As flores também foram coletadas nos mesmos pomares da coleta de frutos, amostrando-se a parte proximal e basal das flores. Foi realizado o teste $\mathrm{F}$ e, quando necessário, aplicado o teste de Scott-Knott a 5\%. Verificou-se que, de maneira geral, há diferenças nutricionais para flores e frutos em relação ao tipo de poda empregada, sendo que a poda drástica proporciona maiores teores de nutrientes, em comparação com a poda contínua. Em relação à parte amostrada observou-se, principalmente para os frutos, que há diferenças entre a parte proximal e distal, bem como para os diferentes estádios de coleta dos frutos.

Palavras-chave: Psidium guajava, estado nutricional, goiaba.

\begin{abstract}
A better understanding of the differences between the levels of nutrients, depending on the type of pruning used in the cultivation of the guava tree, may allow a more adequate understanding of the physiological processes of this fruit. The analysis of flowers is a tool that can be used to assist in assessing the nutritional status of crops, especially perennials. We evaluated the effects of different types of pruning on nutrient concentrations in flowers and fruit, at different developmental stages and in different parts of the fruit. The study was carried out in Vista Alegre do Alto, in orchards of guava variety Paluma. Flowers and fruit were collected in orchards, one under heavy pruning and the other with continuous pruning. The fruit were collected in two stages (two millimeters length and mature) and divided into basal part and apex, with the top toward the stalk. Flowers were collected in the same orchards as the fruits, sampling the basal part and apex of the flowers. F tests were performed and, when necessary, the Scott-Knott test at $\alpha=5 \%$. Overall, there were nutritional differences among flowers and fruits in relation to the type of pruning employed; drastic pruning provided higher levels of nutrients compared with continuous pruning. In relation to the portion of the samples, especially for fruit, there were differences between the apex and base, as well as between different stages of fruit collection.
\end{abstract}

Key words: Psidium guajava, nutritional state, guava.

\section{Introdução}

A goiaba encontra-se em posição de destaque entre as frutas nativas, sendo uma das mais cultivadas no Brasil, o que demonstra sua grande importância, seja para o consumo in natura, seja para o processamento industrial. Os fruticultores utilizam-se de três sistemas de produção para manejar a cultura da goiaba, de acordo com o destino das frutas. O sistema de produção de goiabas "de mesa" visa a

\footnotetext{
Pesquisador Embrapa/CNPC, henrique@cnpc.embrapa.br * - autor correspondente.

Professor Doutor Unesp - Campus Experimental de Registro, danilorozane@ registro.unesp.br

Doutoranda - USP/FZEA, lilianeromualdo@yahoo.com.br

Professor Adjunto Unesp/FCAV, natale@fcav.unesp.br
}

Fecha de Recepción: 18 Octubre, 2010.

Fecha de Aceptación: 8 Junio, 2012. 
atender exclusivamente o mercado para consumo da fruta in natura; o sistema para "indústria" quando o destino dos frutos é a industrialização; e o sistema de "cultivo misto" quando os frutos possuem dupla finalidade, atendendo aos dois mercados simultaneamente. A goiabeira cultivar Paluma é considerada de dupla aptidão, sendo a mais utilizada para atender a este último sistema, podendo ser uma vantagem para os produtores, uma vez que os frutos de melhor qualidade são destinados ao mercado para consumo in natura, alcançando melhores preços, e os demais frutos são destinados ao processamento industrial, gerando produtos diversos (Rozane et al., 2009a).

Tendo em vista tais sistemas, o goiabicicultor pode adotar dois manejos de poda: a drástica e a contínua. A poda de frutificação 'drástica' dos ramos da goiabeira torna-se a via mais importante para favorecer o desenvolvimento dessa frutífera e, por sua vez, o florescimento, possibilitando a colheita de frutos nas épocas desejadas pelo produtor, principalmente nos períodos de menor oferta da fruta no mercado (Rozane et al., 2009a). Empregando o manejo de poda contínua, a colheita, a adubação e a poda são realizadas durante o ano todo. Nesse caso, a planta abriga todos os estágios reprodutivos, desde botão até frutos graúdos.

Um melhor conhecimento das diferenças entre os teores de nutrientes, em função do tipo de poda empregada na cultura da goiaba, pode permitir melhor compreensão dos processos fisiológicos nessa frutífera.

A análise de flores tem sido aplicada com sucesso no diagnóstico de desordens nutricionais em plantas perenes, como frutíferas, cujas folhas se desenvolvem após a floração. A avaliação precoce do estado nutricional, por meio da análise de flores, pode ser de grande valia, pois, possibilita iniciar os ajustes no programa de adubação exatamente no início da estação de crescimento vegetativo, antes que ocorram perdas irreversíveis na produtividade e na qualidade dos frutos. Além disso, sendo as flores órgãos de curta duração, nas quais não ocorrem reações metabólicas tão complexas quanto nas folhas, as mesmas não apresentam diferenças acentuadas entre o teor total do nutriente e a fração ativa fisiologicamente (Martinez et al., 2008).

Além disso, as diferenças nos teores dos elementos em alguns estágios de frutificação podem auxiliar no entendimento de distúrbios nutricionais. Assim, a análise de flores é uma ferramenta que pode ser utilizada para auxiliar na avaliação do estado nutricional das culturas, principalmente perenes.

As avaliações em flores e frutos permitem que importantes reflexões sejam realizadas a cerca das adubações empregadas, pois, a dinâmica dos nutrientes é muito variável, sendo influenciada pelos manejos adotados.

Malavolta et al. (2002) estudaram a repartição de nutrientes nos ramos, folhas e flores na antese da primeira fase reprodutiva do cafeeiro (Coffea arabica L.). As flores dessa planta constituem um forte dreno de nutrientes, variável entre cultivares; a extração total de magnésio $(\mathrm{Mg})$ pelas flores das cultivares representa 52\% do extraído pelas partes da planta (flores, folhas e ramos), o que sugere que a aplicação desse elemento deve ser iniciada antes do florescimento do cafeeiro.

Com base no exposto, objetivou-se avaliar os efeitos de diferentes tipos de poda nos teores de nutrientes em flores e, em frutos em alguns estádios de desenvolvimento e em diferentes partes das goiabas.

\section{Material e Métodos}

O estudo foi realizado em dois pomares comerciais de goiabeiras 'Paluma', com sete anos, propagadas vegetativamente (espaçamento de $7 \mathrm{x}$ $5 \mathrm{~m}$ ). As áreas foram irrigadas por microaspersores tipo bailarina e monitoradas por tensiometria $(60 \%$ da capacidade de campo). Os pomares estão localizados na maior região produtora de goiabas do estado de São Paulo, Brasil, no município de Vista Alegre do Alto, com coordenadas geográficas $21^{\circ}$ 08' Sul, 48 30' Oeste e altitude de $603 \mathrm{~m}$. Segundo a classificação de Köppen, o clima local é do tipo Cwa subtropical com inverno curto, moderado e seco, verão quente e chuvoso, caracterizando duas estações distintas. O solo do pomar foi classificado como Argissolo Vermelho-Amarelo distrófico, textura arenosa/média (Embrapa, 1999).

Cada pomar foi manejado com um tipo de poda: contínua ou drástica. A descrição desses dois manejos de poda pode ser encontrada em Rozane et al. (2009a). A adubação e correção do solo nos pomares avaliados foram realizadas segundo recomendações de Natale et al. (1996) e Natale et al. (2007).

Em ambos os pomares foram coletadas flores durante o pleno florescimento da cultura, no estágio F:65 (Salazar et al., 2006) e, posteriormente, nos mesmos pomares, foram coletados frutos com três 
centímetros de diâmetro (chumbinho) e, também, frutos maduros, no estágio I1:78 e K:89, respectivamente (Salazar et al., 2006).

No momento da coleta das flores, no estádio F:65 (Salazar et al., 2006), foi também realizada a coleta de folhas para a diagnose do estado nutricional das plantas, amostrando-se em cada um dos pomares, $\mathrm{o} 3^{\circ}$ par de folhas recém-maduras, na altura mediana das árvores, nos quatro quadrantes das plantas, segundo indicações de Natale et al. (1996). Foram coletados 40 pares de folhas, como recomendam Rozane et al. (2009b).

Das flores eliminaram-se as pétalas, sendo divididas ao meio, em corte transversal, em duas porções: proximal (voltada para o pedúnculo) e distal. Assim, o delineamento utilizado no estudo foi em blocos casualizados, em esquema fatorial $2 \times 2$ (dois tipos de poda e duas partes das flores) com cinco repetições, sendo coletadas oito flores por planta, duas em cada quadrante, e em três plantas por parcela, totalizando 480 flores.

Os frutos no estágio I1:89 (chumbinho), se encontram em pleno crescimento, atingindo em torno de $80 \%$ do tamanho final. Quando os frutos estão no estágio K:89 (maduros), alcançam o volume final, apresentando coloração amarela (Salazar et al., 2006).

Os frutos coletados nos estádios I1:78 e K:89 (chumbinho e maduro, respectivamente), foram divididos em corte transversal, nas porções: proximal (voltada para o pedúnculo) e distal. O delineamento utilizado no estudo foi em blocos casualizados, em esquema fatorial $2 \times 2 \times 2$ (dois tipos de poda, dois estádios de desenvolvimento dos frutos e duas partes dos frutos, respectivamente) com três repetições, sendo coletados quatro frutos por planta, um em cada quadrante, e em três plantas por parcela, totalizando 288 frutos.

As flores e os frutos, após serem lavados, foram analisados como recomendado para a determinação de nutrientes no tecido vegetal (Bataglia et al., 1983).
Os resultados, depois de tabelados e submetidos ao teste $\mathrm{F}$, foram analisados qualitativamente empregando-se o teste de Scott-Knott (5\%), com auxílio do programa estatístico SISVAR (Ferreira, 2008).

\section{Resultados e Discussão}

Na Tabela 1 são apresentados os teores foliares de nutrientes em cada um dos pomares de goiabeira, em função dos diferentes manejos de poda, com o intuito de se verificar o estado nutricional dos pomares.

O Mg e o zinco ( $\mathrm{Zn}$ ), em ambos os sistemas de poda, e o cobre $(\mathrm{Cu})$ na poda contínua, apresentaram teores foliares abaixo dos preconizados como adequado por Natale et al. (1996) para goiabeira 'Paluma'. Já o manganês (Mn) na poda contínua apresentou teores superiores aos indicados pelos mesmos autores (Tabela 1). Os demais nutrientes se encontram dentro das faixas consideradas adequadas (Natale et al., 1996), indicando o bom estado nutricional dos pomares. Segundo Natale et al. (1996) os teores adequados para goiabeira em macro e micronutrientes são: N: 20-23; P: 1,4-1,8; K: 14-17; Ca: 7-11; Mg: 3,4-4,0; S: 2,5-3,5 $\mathrm{g} \mathrm{kg}^{-1}$ e B: 20-25; Cu: 20-40; Fe: 60-90; Mn: 40-80 e Zn: $25-35 \mathrm{mg} \mathrm{kg}^{-1}$, respectivamente.

Na Tabela 2 pode-se observar que houve resultado significativo somente para tipos de poda para as variáveis nitrogênio $(\mathrm{N})$, potássio $(\mathrm{K}), \mathrm{Cu}$ e Mn. À exceção do Mn a poda drástica apresentou maiores teores de $\mathrm{N}, \mathrm{K}$ e $\mathrm{Cu}$ nas flores no estágio F:65 (Tabela 2). O maior teor de Mn nas flores do pomar submetido à poda contínua pode ser justificado pelo maior teor foliar deste nutriente nas plantas (Tabela 1). Para as diferentes partes das flores, observa-se que houve significância apenas para cálcio $(\mathrm{Ca})$ e boro $(\mathrm{B})$, sendo que em ambos os casos a região proximal apresentou os maiores teores (Tabela 2). Vale salientar que a

Tabela 1. Teores de nutrientes nas folhas de goiabeiras 'Paluma', em função do tipo de poda empregado.

\begin{tabular}{|c|c|c|c|c|c|c|c|c|c|c|c|}
\hline Poda & $\mathrm{N}$ & $\mathrm{P}$ & $\mathrm{K}$ & $\mathrm{Ca}$ & $\mathrm{Mg}$ & $S$ & $\mathrm{~B}$ & $\mathrm{Cu}$ & $\mathrm{Fe}$ & $\mathrm{Mn}$ & $\mathrm{Zn}$ \\
\hline & \multicolumn{7}{|c|}{---------- $\mathrm{g} \mathrm{kg}^{-1}$-------- } & \multicolumn{4}{|c|}{------ $\mathrm{mg} \mathrm{kg}^{-1}$------- } \\
\hline Drástica & 21,0 & 1,7 & 15,8 & 8,4 & 2,2 & 2,3 & 26 & 22 & 76 & 55 & 19 \\
\hline Contínua & 21,4 & 1.8 & 15,4 & 9,7 & 2.7 & 2,4 & 27 & 10 & 100 & 133 & 20 \\
\hline
\end{tabular}


Tabela 2. Resumo da análise de variância para diferentes tipos de poda e partes das flores no estágio F:65.

\begin{tabular}{|c|c|c|c|c|c|c|c|c|c|c|c|}
\hline Poda (P) & $\mathrm{N}$ & $\mathrm{P}$ & $\mathrm{K}$ & $\mathrm{Ca}$ & $\mathrm{Mg}$ & $S$ & B & $\mathrm{Cu}$ & $\mathrm{Fe}$ & $\mathrm{Mn}$ & $\mathrm{Zn}$ \\
\hline & \multicolumn{6}{|c|}{ - } & \multicolumn{5}{|c|}{----------- $\mathrm{mg} \mathrm{kg}^{-1}$------------- } \\
\hline Drástica & $19,5 \mathrm{a}$ & 2,4 & $26,2 \mathrm{a}$ & 10,6 & 3,2 & 2,3 & 23 & $14 \mathrm{a}$ & 45 & $44 b$ & 31 \\
\hline Contínua & $16,8 \mathrm{~b}$ & 2,2 & $20,2 b$ & 10,0 & 3,3 & 2,0 & 24 & $9 \mathrm{~b}$ & 50 & $102 \mathrm{a}$ & 31 \\
\hline Teste F & $19,51^{* *}$ & $1,70^{\mathrm{ns}}$ & $18,1^{* *}$ & $0,51^{\mathrm{ns}}$ & $0,22^{\mathrm{ns}}$ & $4,10^{\mathrm{ns}}$ & $1,10^{\mathrm{ns}}$ & $42,73^{* *}$ & $3,75^{\mathrm{ns}}$ & $50,41^{* *}$ & $0,02^{\text {ns }}$ \\
\hline \multicolumn{12}{|l|}{$1(\mathrm{PF})$} \\
\hline Proximal & 18,9 & 2,3 & 22,1 & $11,5 \mathrm{a}$ & 3,3 & 2,2 & $26 \mathrm{a}$ & 10 & 49 & 72 & 27 \\
\hline Distal & 17,5 & 2,3 & 24,3 & $9,2 \mathrm{~b}$ & 3,1 & 2,1 & $21 \mathrm{~b}$ & 12 & 46 & 73 & 35 \\
\hline Teste F & $5,39^{\mathrm{ns}}$ & $0,69^{\text {ns }}$ & $2,51^{\mathrm{ns}}$ & $7,40^{*}$ & $0,39^{\text {ns }}$ & $0,57^{\mathrm{ns}}$ & $11,40^{* *}$ & $4,11^{\mathrm{ns}}$ & $1,12^{\mathrm{ns}}$ & $0,01^{\mathrm{ns}}$ & $2,94^{\mathrm{ns}}$ \\
\hline $\mathrm{P} \times \mathrm{PF}$ & $0,88^{\mathrm{ns}}$ & $1,70^{\mathrm{ns}}$ & $0,93^{\mathrm{ns}}$ & $0,01^{\mathrm{ns}}$ & $0,39^{\text {ns }}$ & $0,57^{\mathrm{ns}}$ & $0,66^{\mathrm{ns}}$ & $2,49^{\mathrm{ns}}$ & $0,07^{\mathrm{ns}}$ & $0,08^{\mathrm{ns}}$ & $2,04^{\mathrm{ns}}$ \\
\hline CV (\%) & 5,8 & 10,5 & 10,5 & 14,5 & 11,5 & 10,5 & 10,5 & 11,6 & 10,9 & 19,5 & 13,1 \\
\hline
\end{tabular}

1 Porções florais, **; *; ns: significativo pelo teste $\mathrm{F}$ a $1 \%$; $5 \%$ e não significativo, respectivamente. Médias seguidas de letras iguais, na vertical, não diferem pelo teste de Scott-Knott a $5 \%$ de probabilidade.

região proximal está voltada para o pedúnculo e, os nutrientes cálcio e boro são considerados imóveis nas plantas. Veríssimo et al. (2006) encontraram resultados semelhantes em pereira, cujos teores de $\mathrm{Ca}$ e B foram superiores na porção proximal em relação ao ápice de flores e frutos.

A ocorrência de "bitter pit" na maçã cultivar Gala é consequência de baixos teores de Ca na casca e na polpa, e de altos teores de K na casca; desse modo, a amostragem da casca é mais indicada para a análise nutricional visando a caracterizar a ocorrência de "bitter pit" em maçãs 'Gala' (Amarante et al., 2006).

Assim, devido a ambos os elementos apresentarem pouca mobilidade, era de se esperar a baixa redistribuição, ocorrendo os teores mais elevados na região proximal dos frutos.

O B é essencial para a germinação dos grãos de pólen e o crescimento do tubo polínico, além do metabolismo fenólico e protéico, integridade e funcionamento das membranas celulares. Desempenha, também, importante papel na migração e metabolismo de carboidratos, facilitando o transporte dos açúcares através das membranas, na forma do complexo açúcar-borato (Malavolta et al., 1997). Em estudo avaliando flores para diagnose do estado nutricional de B em maçãs, Wojcik (2002) concluiu que as flores podem ser usadas para a predição e recomendação de adubos. O Ca atua na síntese de novas paredes celulares, no fuso mitótico, na atuação normal das membranas e como mensageiro secundário para um número de respostas das plantas para sinais ambientais e hormonais (Taiz e Zeiger, 2009).

Malavolta et al. (2006), em avaliação da redistribuição de nutrientes em laranjeira Natal, encontraram para Ca que os maiores teores estão presentes nas flores e, para boro, o mesmo se encontra distribuído de maneira equivalente nas folhas, flores e frutos.

Um dos objetivos de quantificar os teores macro e micronutrientes em nível de flores está na possibilidade de utilizar essas informações no esclarecimento de distúrbios fisiológicos, como o abortamento florais (Veríssimo et al., 2006) e, ou mesmo, de frutos.

Matinez et al. (2003) citam que a utilização de flores para predição do estado nutricional é vantajosa devida à rápida diagnose e boa precisão. Os autores trabalharam com vários talhões de cafeeiro e verificaram que há menor valor do coeficiente de variação quando se trabalham com flores ao invés de folhas. Em ensaio com Oliveira, Khelil et al. (2010) também recomendam a utilização das flores para avaliação da diagnose nutricional.

Verifica-se na Tabela 3 que não houve significância para a interação entre os três fatores e, também, para a interação entre a poda e as partes do fruto. Na interação entre tipo de fruto e poda houve diferença estatística para $\mathrm{N}$, fósforo $(\mathrm{P}), \mathrm{K}$, $\mathrm{Ca}, \mathrm{Mg}$, enxofre (S), Cu e, para a interação tipo de fruto e porção do fruto houve resultado significativo apenas para o $\mathrm{Mn}$. Os micronutrientes $\mathrm{B}$, ferro $(\mathrm{Fe})$ e zinco (Zn) não apresentaram resultados significativos para a interação.

O estágio de crescimento dos frutos apresentou resultado significativo para todos os nutrientes, acarretando ao fruto maduro sempre o menor teor. Tal resultado pode ser devido ao efeito diluição que ocorre com o desenvolvimento e, o consequente amadurecimento do fruto. 
Tabela 3. Resumo da análise de variância para diferentes tipos de poda, estádios de frutos (I1:78 e K:89) e porções de frutos.

\begin{tabular}{|c|c|c|c|c|c|c|c|c|c|c|c|}
\hline Fruto $(F)$ & $\mathrm{N}$ & $\mathrm{P}$ & $\mathrm{K}$ & $\mathrm{Ca}$ & $\mathrm{Mg}$ & $\mathrm{S}$ & B & $\mathrm{Cu}$ & $\mathrm{Fe}$ & $\mathrm{Mn}$ & $\mathrm{Zn}$ \\
\hline & & & & & & ------ & -- & - & $\mathrm{mg} \mathrm{kg}$ & & ---- \\
\hline I1:78 & $9,8 \mathrm{~b}$ & $1,2 \mathrm{~b}$ & $16,6 b$ & $0,9 b$ & $0,6 \mathrm{~b}$ & $1,0 \mathrm{~b}$ & $10 \mathrm{~b}$ & $7 \mathrm{~b}$ & $23 b$ & $8 \mathrm{~b}$ & $15 b$ \\
\hline K:89 & $14,1 \mathrm{a}$ & $1,8 \mathrm{a}$ & $22,8 \mathrm{a}$ & $1,7 \mathrm{a}$ & $1,2 \mathrm{a}$ & $1,6 \mathrm{a}$ & $12 \mathrm{a}$ & $10 \mathrm{a}$ & $31 \mathrm{a}$ & $16 \mathrm{a}$ & $21 \mathrm{a}$ \\
\hline Teste F & $101,73^{* *}$ & $208,29^{* *}$ & $174,60^{* *}$ & $94,73^{* *}$ & $175,30^{* *}$ & $317,59^{* *}$ & $51,04^{*}$ & $20,54^{* * *}$ & $34,31^{* *}$ & $128,54^{* *}$ & $62,12^{* *}$ \\
\hline \multicolumn{12}{|l|}{ Poda (P) } \\
\hline Drástica & $13,6 \mathrm{a}$ & $1,6 a$ & $21,4 \mathrm{a}$ & $1,4 \mathrm{a}$ & 0,9 & $1,4 \mathrm{a}$ & 11 & $10 \mathrm{a}$ & $30 \mathrm{a}$ & $10 \mathrm{~b}$ & $20 \mathrm{a}$ \\
\hline Contínua & $10,8 \mathrm{~b}$ & $1,5 b$ & $18,0 \mathrm{~b}$ & $1,1 \mathrm{~b}$ & 0,8 & $1,1 b$ & 11 & $7 \mathrm{~b}$ & $25 b$ & $14 \mathrm{a}$ & $17 \mathrm{~b}$ \\
\hline Teste F & $57,96^{* *}$ & $19,29^{* *}$ & $52,06^{* *}$ & $16,44^{* *}$ & $4,08^{\mathrm{ns}}$ & $93,59^{* *}$ & $0,05^{\mathrm{ns}}$ & $10,48^{* *}$ & $19,61^{* *}$ & $18,21^{* *}$ & $15,53^{* *}$ \\
\hline \multicolumn{12}{|l|}{${ }^{1}(\mathrm{PF})$} \\
\hline Proximal & $12,9 \mathrm{a}$ & $1,6 \mathrm{a}$ & 19,8 & $1,1 \mathrm{~b}$ & $1,0 \mathrm{a}$ & $1,3 \mathrm{a}$ & 10 & 8 & 27 & $13 \mathrm{a}$ & 19 \\
\hline Distal & $11,0 \mathrm{~b}$ & $1,5 \mathrm{~b}$ & 19,6 & $1,4 \mathrm{a}$ & $0,7 b$ & $1,2 \mathrm{~b}$ & 12 & 8 & 27 & $11 \mathrm{~b}$ & 18 \\
\hline Teste F & $21,08^{* *}$ & $12,64^{* *}$ & $0,22^{\mathrm{ns}}$ & $8,06^{*}$ & $42,35^{* *}$ & $21,00^{* *}$ & $0,89^{\text {ns }}$ & $0,18^{\mathrm{ns}}$ & $0,32^{\text {ns }}$ & $7,86^{*}$ & $1,93^{\mathrm{ns}}$ \\
\hline $\mathrm{F} \times \mathrm{P}$ & $11,12^{* *}$ & $23,14^{* *}$ & $16,32^{* *}$ & $4,97^{*}$ & $11,33^{* *}$ & $25,92^{* *}$ & $0,01^{\mathrm{ns}}$ & $15,09^{* *}$ & $0,32^{\text {ns }}$ & $2,51^{\mathrm{ns}}$ & $3,44^{\mathrm{ns}}$ \\
\hline $\mathrm{F} \times \mathrm{PF}$ & $3,85^{\text {ns }}$ & $0,04^{\mathrm{ns}}$ & $0,09^{\text {ns }}$ & $2,01^{\mathrm{ns}}$ & $2,46^{\mathrm{ns}}$ & $1,03^{\mathrm{ns}}$ & $3,88^{\mathrm{ns}}$ & $0,05^{\mathrm{ns}}$ & $2,47^{\mathrm{ns}}$ & $6,55^{*}$ & $0,05^{\mathrm{ns}}$ \\
\hline $\mathrm{P} \times \mathrm{PF}$ & $0,34^{\mathrm{ns}}$ & $2,14^{\mathrm{ns}}$ & $0,38^{\mathrm{ns}}$ & $0,04^{\mathrm{ns}}$ & $0,45^{\mathrm{ns}}$ & $1,03^{\mathrm{ns}}$ & $4,47^{\mathrm{ns}}$ & $1,16^{\mathrm{ns}}$ & $0,02^{\mathrm{ns}}$ & $1,20^{\mathrm{ns}}$ & $0,86^{\mathrm{ns}}$ \\
\hline $\mathrm{F} \times \mathrm{P} \times \mathrm{PF}$ & $0,01^{\mathrm{ns}}$ & $0,39^{\text {ns }}$ & $3,97^{\mathrm{ns}}$ & $0,16^{\mathrm{ns}}$ & $1,25^{\mathrm{ns}}$ & $0,25^{\mathrm{ns}}$ & $1,92^{\text {ns }}$ & $0,18^{\mathrm{ns}}$ & $2,05^{\mathrm{ns}}$ & $1,20^{\mathrm{ns}}$ & $0,48^{\text {ns }}$ \\
\hline $\mathrm{CV}(\%)$ & 8,7 & 6,2 & 5,9 & 16,0 & 10,0 & 6,3 & 25,0 & 21,8 & 10,4 & 13,6 & 9,4 \\
\hline
\end{tabular}

1 Porções de fruto, ${ }^{*} ;$; * ns: significativo pelo teste $\mathrm{F}$ a $1 \% ; 5 \%$ e não significativo, respectivamente. Médias seguidas de letras iguais, na vertical, não diferem pelo teste de Scott-Knott a $5 \%$ de probabilidade.

Para tipos de poda, as variáveis $\mathrm{N}, \mathrm{P}, \mathrm{K}, \mathrm{Ca}$, $\mathrm{S}, \mathrm{Cu}, \mathrm{Fe}, \mathrm{Mn}$ e Zn apresentaram significância em função dos manejos de poda empregados, sendo que, exceto para Mn, a poda drástica promoveu maiores teores dos demais elementos nos frutos da goiabeira. No caso do manganês, a explicação está no maior teor foliar deste nutriente apresentado pela planta (Tabela 1), a exemplo do que também ocorreu nas flores (Tabela 2).

Da mesma maneira que para as flores, o mesmo raciocínio pode ser feito para os frutos; a demanda de nutrientes é maior quando as goiabeiras são podadas de forma drástica, devido à grande exigência de elementos para a formação dos frutos, em um curto espaço de tempo. No caso da poda contínua, a qual apresenta todas as fenofases em uma mesma planta de goiabeira (crescimento vegetativo, gemas florais, flores e frutos), o requerimento de nutrientes para a formação dos frutos é menor, quando comparado à poda drástica. Isso deve influenciar no manejo da adubação, visto as maiores quantidades de elementos que devem ser destinados à formação dos frutos, em uma mesma fase.
Para as porções do fruto, $\mathrm{N}, \mathrm{P}, \mathrm{Ca}, \mathrm{Mg}, \mathrm{S}$ e $\mathrm{Mn}$ apresentaram diferenças significativas entre as partes amostradas (Tabela 3). N, P, Mg, S e Mn ocorreram em maiores teores na parte baixeira do fruto, a qual está oposta ao pedúnculo. Já para o cálcio, o resultado foi contrário aos dos demais nutrientes, em que a parte de cima, ou aquela ligada ao pedúnculo, apresentou maiores valores, corroborando os resultados encontrados para flores (Tabela 2), aliados à imobilidade do nutriente na planta.

Em avaliação de características de produção e de qualidade dos frutos da goiabeira 'Paluma', produzidos em plantas com e sem irrigação, submetidas a diferentes intensidades de poda de frutificação, em diferentes épocas do ano, Serrano et al. (2007) afirmam que as boas produções observadas podem estar associadas ao clima favorável e ao estado nutricional das plantas.

Bouranis et al. (2010) verificaram em amendoeiras que as flores podem ser usadas para a avaliação da dinâmica dos nutrientes em folhas e frutos. Pestana et al. (2001) em estudo avaliando a correlação de flores e folhas, flores e frutos em 
Tabela 4. Teores de nitrogênio, fósforo, potássio, cálcio, magnésio, enxofre e cobre em função da interação tipos de frutos e tipos de poda.

\begin{tabular}{lccccccc}
\hline Fruto & $\mathrm{N}$ & $\mathrm{P}$ & $\mathrm{K}$ & $\mathrm{Ca}$ & $\mathrm{Mg}$ & $\mathrm{S}$ & $\mathrm{Cu}$ \\
\hline $\mathrm{I} 1: 78$ & & & & & & & $\mathrm{mg} \mathrm{kg}^{-1}$ \\
Drástica & $16,4 \mathrm{a}^{*}$ & $2,1 \mathrm{a}$ & $25,5 \mathrm{a}$ & $1,9 \mathrm{a}$ & $1,3 \mathrm{a}$ & $1,8 \mathrm{a}$ & $13 \mathrm{a}$ \\
Contínua & $11,8 \mathrm{~b}$ & $1,7 \mathrm{~b}$ & $20,2 \mathrm{~b}$ & $1,4 \mathrm{~b}$ & $1,0 \mathrm{~b}$ & $1,3 \mathrm{~b}$ & $7 \mathrm{~b}$ \\
\hline $\mathrm{K}: 89$ & & & & & & & $6 \mathrm{a}$ \\
\hline Drástica & $10,8 \mathrm{a}$ & $1,3 \mathrm{a}$ & $17,3 \mathrm{a}$ & $0,9 \mathrm{a}$ & $0,6 \mathrm{a}$ & $1,0 \mathrm{a}$ & $7 \mathrm{a}$ \\
Contínua & $9,0 \mathrm{~b}$ & $1,3 \mathrm{a}$ & $15,8 \mathrm{~b}$ & $0,8 \mathrm{a}$ & $0,7 \mathrm{a}$ & $0,9 \mathrm{~b}$ & $\mathrm{~g} \mathrm{k}^{-1}$ \\
\hline
\end{tabular}

* Médias seguidas de letras iguais, na vertical, não diferem pelo teste de Scott-Knott a 5\% de probabilidade.

laranjeiras, concluem que os teores de $\mathrm{P}, \mathrm{Fe}$ e $\mathrm{Mn}$ nas flores podem ser usados para estimar a clorofila nas folhas e, que os teores de $\mathrm{P}, \mathrm{Mg}$ e Mn se correlacionaram positivamente com o tamanho de fruto.

A interação indica para frutos no estágio chumbinho (I1:78) que as plantas podadas de forma drástica apresentam maiores teores de nutrientes, em relação à poda contínua (Tabela 4). Da mesma forma, para os frutos maduros, o N, o K e o S apresentaram diferenças significativas e, novamente, os frutos provenientes de plantas podadas drasticamente apresentaram maiores teores em relação à contínua.

Assim, principalmente para frutos no estádio I1:78, quando ocorre grande demanda de nutriente para a formação dos frutos ou a alocação de reservas, nas plantas podadas de maneira drástica, a exigência nutricional nesta fase parece ser maior. No entanto, quando os frutos já estão formados, ou seja, no estágio maduro, as diferenças diminuem, sendo que apenas para os nutrientes $\mathrm{N}, \mathrm{K}$ e $\mathrm{S}$ a diferença entre podas persiste (Tabela 4).

A consequência da maior extração de nutrientes pelos frutos das goiabeiras submetidas à poda drástica é uma fertilização mais intensa, além do requerimento de um melhor manejo do pomar, afinal, a exportação de elementos também será maior.

Acompanhada da irrigação e da adubação, a poda de frutificação 'drástica' dos ramos da goiabeira, torna-se a via mais importante para favorecer o desenvolvimento dessa frutífera e, por sua vez, o florescimento, possibilitando a colheita de frutos nas épocas desejadas pelo produtor (Rozane et al., 2009a). As exigências nutricionais provocadas pelo manejo dos pomares com poda drástica são tão intensas que Silva et al. (2009) elaboraram um software para a realização da calagem e da adubação das plantas conduzidas neste sistema. Segundo Serrano et al.
(2008) a poda curta ou drástica proporciona menor número de frutos por ramo e menor pegamento de frutos, em goiabeira 'Paluma'.

A Tabela 5 apresenta os teores de nutrientes em função da interação estágios de crescimento dos frutos e partes dos frutos.

Quando se avaliou os teores de Mn nos frutos no estádio I1:78, observou-se maiores teores na porção proximal; contudo, com o amadurecimento do fruto, no estágio K:89, esta diferença não mais ocorreu (Tabela 5). Matias et al. (2006) avaliaram diferentes épocas e folhas de coqueiro para avaliação do estado nutricional do coqueiro anão, e constataram resultados diferentes para os nutrientes avaliados nas épocas e nas folhas estudadas.

Tabela 5. Teores de manganês no fruto em função da interação estágios e partes dos frutos.

\begin{tabular}{lc}
\hline Fruto & $\mathrm{Mn}$ \\
\hline I1:78 & $\mathrm{mg} \mathrm{kg}^{-1}$ \\
Proximal & $18 \mathrm{a}^{*}$ \\
Distal & $14 \mathrm{~b}$ \\
\hline K:89 & $8^{\mathrm{a}}$ \\
\hline Proximal & $9^{\mathrm{a}}$ \\
Distal & \\
\hline
\end{tabular}

* Médias seguidas de letras iguais, na vertical, não diferem pelo teste de Scott-Knott a $5 \%$ de probabilidade.

\section{Conclusões}

Os resultados apresentados permitem inferir que há diferenças na demanda e na alocação de nutrientes pelos frutos em pomares submetidos a diferentes tipos de poda. 
A poda drástica induziu maiores teores de nutrientes nos frutos, em relação à poda contínua.

A região proximal dos frutos apresentou maiores teores de nutrientes, em relação à distal.

\section{Agradecimentos}

À Indústria de Polpas e Conservas VAL Ltda. pela disponibilização da área.

\section{Literatura Citada}

Amarante, C.V.T.; Chaves, D.V.; Ernani, P.R.

2006 Análise multivariada de atributos nutricionais associados ao "bitter pit" em maçãs 'Gala'. Pesquisa Agropecuária Brasileira, v. 41, n. 5, pp. 841-846.

Bataglia, O.C.; Furlani, A.M.C.; Teixeira, J.P.F.; Furlani, P. R.; Gallo, J.R.

1983 Métodos de análise química de plantas. Campinas: Instituto Agronômico, 48 p. (Boletim Técnico, 78).

Bouranis, D.L.; Chorianopoulou, S.N.; Zakynthinos, G.; Sarlis, G.; Drossopoulos, J.B.

2001 Flower analysis for prognosis of nutritional dynamics of almond tree. Journal of Plant Nutrition, v. 24, n. 4, pp. 705-716.

Embrapa. Empresa Brasileira de Pesquisa Agropecuária. 1999 Sistema Brasileiro de classificação de solos. Rio de Janeiro: Embrapa, 412 p.

Ferreira, D.F. 2008 Sisvar: um programa para análises e ensino estatístico. Revista Científica Symposium, v. 6, n. 2, pp. 36-41.

Khelil, M.B.; Sanaa, M.; Msallem, M.; Larbi, A.

2010 Floral analysis as a new approach to evaluate the nutritional status of olive trees. Journal of Plant Nutrition, v. 33, n. 5, pp. 627-639, 2010.

Malavolta, E.; Vitti, G.C.; Oliveira, S.A. 1997 Avaliação do estado nutricional das plantas: princípios e aplicações. Piracicaba: POTAFOS, 319 p.

Malavolta, E.; Leão, H.C.; Oliveira, S.C.; Lavres Júnior, J.; Moraes, M.F.; Cabral, C.P.; Malavolta, M.

2002 Repartição de nutrientes nos ramos, folhas e flores do cafeeiro. Pesquisa Agropecuária Brasileira, v. 37, n. 7, pp. 1017-1022.

Matias, S.S.R.; Aquino, B.F.; Freitas, J.A.D.; Hernandez, F.F.F. 2006 Análise foliar de coqueiro anão em duas épocas diferentes em relação a doses de nitrogênio e potássio. Revista Ciência Agronômica, v. 37, n. 3, pp. 264-269.

Martinez, H.E.P. Zabini, A.V.; Neves, Y.P.; Clemente, J.M.; Pedrosa, A.W.

2008 Diagnose foliar em cafeeiro. In: Prado, R.M.; Rozane, D.E.; Vale, D.W.; Correia, M.A.R.; Souza, H.A. Nutrição de plantas diagnose foliar em grandes culturas. Jaboticabal: FCAV, Capes/FUNDUNESP, pp. 267-301.

Martinez, H.E.P.; Souza, R.B.; Bayona, J.A.; Alvarez V., V.H.; Sanz, M.

2003 Coffee-tree floral analysis as a mean of nutritional diagnosis. Journal of Plant Nutrition, v. 26, n. 7, pp. 1467-1482.

Natale, W. Coutinho, E.L.M.; Boaretto, A.E.; Pereira, F.M. 1996 Goiabeira: calagem e adubação. Jaboticabal: FUNEP, $22 \mathrm{p}$.
Natale, W. Prado, R.M.; Rozane, D.E.; Romualdo, L.M. 2007 Efeitos da calagem na fertilidade do solo e na nutrição e produtividade da goiabeira. Revista Brasileira de Ciência do Solo, v. 31, n. 6, pp. 1475-1485.

Pestana, M.; Correia, P. J.; Varennes, A.; Abadía, J.; Faria, E. A. 2001 The use of floral analysis to diagnose the nutritional status of orange trees. Journal of Plant Nutrition, v. 24, n. 12, pp. 1913-1923.

Rozane, D.E.; Brugnara, V.; Souza, H.A.; Amorim, D.A. 2009a Condução, arquitetura e poda da goiabeira para 'mesa' e/ou 'indústria'. In: Natale, W.; Rozane, D.E.; Souza, H.A.; Amorim, D.A. Cultura da goiaba do plantio à comercialização. Jaboticabal: FCAV/Capes/CNPq/FAPESP/Fundunesp/ SBF, v. 2. pp. 429-470.

Rozane, D.E.; Natale, W.; Prado, R.M.; Barbosa, J.C.

2009b Tamanho da amostra foliar para avaliação do estado nutricional de goiabeiras com e sem irrigação. Revista Brasileira de Engenharia Agrícola e Ambiental, v. 13, n. 3, pp. 233-239.

Salazar, D.M.; Melgarejo, P.; Martínez, R.; Martínez, R.R.; Hernández, F.; Burguera, M.

2006 Phenological stages of the guava tree (Psidium guajava L.). Scientia Horticultare, v. 108, n. 2, pp. 157-161.

Serrano, L.A.L.; Marinho, C.S.; Ronchi, C.P.; Lima, I.M.; Martins, M.V.V.; Tardin, F.D.

2007 Goiabeira 'Paluma'sob diferentes sistemas de cultivo, épocas e intensidades de poda de frutificação. Pesquisa Agropecuária Brasileira, v. 42, pp. 785-792.

Serrano, L.A.L.; Marinho, C.S.; Lima, I.M.; Martins, M.V.V.; Ronchi, C.P.; Tardin, F.D.

2008 Fenologia da goiabeira 'Paluma'sob diferentes sistemas de cultivo, épocas e intensidades de poda de frutificação. Bragantia, v. 67, n. 3, pp. 701-7012.

Silva, S.H.M.G.; Natale, W.; Santos, E.D.H.; Bendini, H.N. 2009 Fert-goiaba: software para recomendação de calagem e adubação para goiabeira cultivar paluma, irrigada e manejada com poda drástica. In: Natale, W.; Rozane, D.E.; Souza, H.A.; Amorim, D.A. Cultura da goiaba do plantio à comercialização. Jaboticabal: FCAV/Capes/CNPq/FAPESP/ Fundunesp/ SBF, v. 2. pp. 281-284.

Veríssimo, V.; Herter, F.G.; Rodrigues, A.C.; Trevisan, R.; Marafon, A.C.

2006 Níveis de cálcio e boro de gemas florais de pereira (Pyrus sp.) no Sul do Brasil. Revista Brasileira de Fruticultura, v. 28, n. 1, pp. 28-31.

Taiz, L.; Zeiger, E.

2009 Fisiologia Vegetal. Porto Alegre: ARTMED, 820 p.

Wojcik, P.

2002 Boron analysis in tissues before apple tree bloom can be used to asses boron nutritional status. Journal of Plant Nutrition, v. 25, n. 5, pp. 1011-1020. 
